\title{
Multihazard weather risk perception and preparedness in eight countries
}

\begin{abstract}
Weather risk perception research lacks multihazard and transcultural datasets. This hypothesis-generating study used a cognitive behavioral approach and Brunswik's lens model for subjective risk parameters across eight countries. In Germany, Poland, Israel, the United States, Brazil, India, Malaysia, and Australia, 812 field interviews took place with a uniform set of 37 questions about weather interest, media access, elementary meteorological knowledge, weather fear, preparedness, loss due to weather, and sociodemography. The local randomized quota samples were strictly tested for sample errors; however, they cannot be considered representative for individual countries due to sample size and methodology. Highly rated subjective risks included flood, heat, tornado, and lightning. Weather fear was most prominent in the Malaysian sample and lowest in the German.
\end{abstract}

Subjective elements were further explored with bivariate correlations and a multivariate regression analysis. Sociodemography correlated with psychological variables like knowledge, interest, and fear. Fear was related with subjective risk; less educated and informed people were more fearful. A linear regression analysis identified interest, gender, housing type, education, loss due to weather, and local weather access as the significant predictors for preparedness. The level of preparedness was highest in the United States and Australia and lowest in the Malaysian and Brazilian samples. A lack of meteorological training and infrequent loss experiences make media communication important and emphasize the value of repetition for basic information. Elements of this survey can serve to monitor weatherrelated psychological orientations of vulnerable population groups. Finally, this survey provides a template with which larger representative transcultural multihazard perception studies can be pursued.

Keyword : Communications/decision making; Education; Emergency preparedness; Risk assessment; Societal impacts 